\title{
Evolution of Antibiotic Resistance in Enterobacter Spp Isolated at the Yaounde University Teaching Hospital from January 2008 to November 2019
}

\author{
Kamga $\mathrm{HG}^{1,2 *}$, Nzukui $\mathbf{N}^{3}$, Lyonga Mbamyah Emilia ${ }^{1,5}$, Tchuedji YGN $^{4,5}$, \\ Betbeui AC ${ }^{1,2}$, Noubom $\mathbf{M}^{6}$, Boum IIY ${ }^{7}$ and Arthur $\mathrm{E}^{2}$ \\ ${ }^{1}$ Department of Microbiology, Parasitology, Haematology and Infectious Diseases, Faculty of \\ Medicine and Biomedical Sciences, University of Yaounde I, Cameroon \\ ${ }^{2}$ Laboratory of Bacteriology, University Teaching Hospital, Cameroon \\ ${ }^{3}$ School of Health Sciences, Catholic University of Central Africa, Cameroon \\ ${ }^{4}$ Department of Microbiology, Laboratory of Microbiology, University of Yaounde I, Cameroon \\ ${ }^{5}$ Department of Public Health, University of Yaounde I, Cameroon \\ ${ }^{6}$ Department of Biological sciences, University of Dschang, Cameroon \\ ${ }^{7}$ Epicenter Africa Research Centre, Cameroon
}

Research Article

Volume 6 Issue 2

Received Date: March 26, 2021

Published Date: April 20, 2021

DOI: $10.23880 /$ oajmb-16000191

*Corresponding author: Hortense Gonsu Kamga, Department of Microbiology, Parasitology, Haematology and Infectious Diseases, Faculty of Medicine and Biomedical Sciences, University of Yaounde I, Yaounde, Bp : 1364, Cameroon, Tel: +237677933270; Email: hgonsu@gmail.com

\section{Abstract}

Purpose: A study was conducted to evaluate the evolution of the resistance of Enterobacter spp to antibiotics during twelve years and to update the data.

Method: A retro-prospective study was carried from January 2008 to November 2019. Data was extracted from the registers of the bacteriology laboratory and the strains from samples received from the different units of the YUTH. The study of the antibiotic resistance profile of these species and phenotypic analysis was carried out by the method of discs diffusion in Mueller-Hinton agar. Phenotypic characterization was carried out by synergy test and modified Hodge test.

Findings: A total of 109 strains were isolated in our study. Enterobacter species showed high resistance with a peak in 2012 for cephalosporins, in 2011 for aminoglycosides, in 2018 for quinolones, in 2019 for carbapenems with the frequencies of $80 \%, 45 \%, 37 \%$ and $36,1 \%$ respectively. These species exhibited $30 \%$ resistance to colistin. The resistance peak to the majority of antibiotics between 2018 and 2019 reflects an increase of resistance. The Extended Spectrum $\bigotimes$ - Lactamases (ESBL) phenotype was the most represented with frequency of $32.4 \%$.

Unique contribution to theory, practice and policy: To Update the data on the evolution of Enterobacter spp, which will help to establish a surveillance strategy in Cameroonand adapt an adequate treatment regimen.

Keywords: Evolution; Enterobacter Spp; Phenotypes; Esbls; Antibiotic Resistance

Abbreviations: CLSI: Clinical Laboratory Standard Institute. 


\section{Open Access Journal of Microbiology \& Biotechnology}

\section{Introduction}

Since their discovery, antibiotics have been the main drug used in the treatment of bacterial infections, in fact antibiotics have been the main defense in the treatment of bacterial infections. However, bacteria are increasingly subject to resistance mechanisms allowing them to escape the actions of antibiotics [1]. Among these bacteria, Enterobacter species are involved in several nosocomial infections [2,3]. Antibiotic resistance is a real public health problem in the world. The spread of resistant pathogenic bacteria results from poor hygiene, inappropriate and abusive use of antibiotics in low income countries [4]. Several strains of Enterobacter are resistant to a majority of antibiotics except to Carbapenems and colistin [5]. Enterobacter species have presented resistance to $\beta$-lactams, quinolones and aminoglycosides in previous studies [6]. Studies of Gonsu, et al. [7] and Nouetchognou, et al. [8] have also revealed resistant strains of Enterobacter in Cameroon. None of these studies have explored the evolution of antibiotic resistance of Enterobacter species.

The level of antibiotic resistance varies from continent to continent, from country to country and from year to year. It seemed important to conduct a retro-prospective study on the evolution of antibiotic resistance of Enterobacter isolated in Cameroon during twelve years.

\section{Methodology}

\section{Ethical Considerations}

This study was granted an ethical clearance by the Catholic University of Central Africa Committee under the number N $N^{\circ} 2019 / 0960 / C E I R S H / E S S / M I M$. Anonymity of participants and confidentiality of results were scrupulously respected.

\section{Clinical Specimens}

The strains were isolated from vaginal secretions, urine, catheter tip, blood, eye secretions, urinary catheter tip analyzed in the bacteriology laboratory of University Teaching Hospital from September to November 2019. For the retrospective study, the data were extracted from the registers from January 2008 to October 2019. These data were sex, age, provenance unit, type of sample, isolated species and results of susceptibility tests.

\section{Identification}

The samples were cultured following standard bacteriological procedures. Identification was performed using the API20E (BioMérieux, France) following the manufacturer's instructions.

\section{Antimicrobial Susceptibility Testing}

Susceptibility tests were doneby the disc diffusion method on Mueller-Hinton agar according to the recommendations of the CASFM [9].

\section{Phenotypic Characterization}

The double disc synergy testwas performed to determine ESBLs phenotypes according to the recommendations of the CASFM [9]. Each isolate identified in this study was tested with ceftazidime, placed about $30 \mathrm{~mm}$ around amoxicillin/ clavulanic acid disc. After 24 hours of incubation, the isolate was considered an ESBL-producer when it exhibited an increase in the zone of inhibition around ceftazidime/ clavulanic acid by $5 \mathrm{~mm}$ or more. The modified Hodge test was performed to determine the presence of carbapenemase according to the recommendations of Clinical Laboratory Standard institute (CLSI) [10]. Mueller-Hinton agar was inoculated with a sensitive strain of $E$. coli ATCC 25922. A disc containing $10 \mu \mathrm{g}$ of carbapenem (meropenem or ertapenem) was placed in the center of the agar, and then the strain of Enterobacter spp was taken from a suspension and seeded on the agar as a line from the disc to the edge of the Petri dish. A positive control (resistance strain) and negative control (sensitive strain) were deposited under the same conditions. The plates were incubated aerobically at $37^{\circ} \mathrm{C}$ for 24 hours. The production of a carbapenemase is characterized by the growth of Enterobacter spp strain towards the antibiotic disc [11]. The search for other resistance phenotypes was based on the results of the susceptibility tests.

\section{Statistical Analysis}

The data was analyzed using SPSS version 2.1and CSPro version 7.1. Graphs were drawed with Microsoft Excel 2016.

\section{Results}

Samples were collected on 54 (49.5\%) males and 55 $(50.5 \%)$ females. The age range of $[60 ; 80]$ years was the most affected with a frequency of $23.9 \%$. Overall 109 strains of Enterobacter were isolated from samples, distributed as follows: Blood $(36.7 \%)$, pus $(25.7 \%)$, urine $(20,2 \%)$, urinary catheter tips $(12,8 \%)$, vaginal secretions $(1,8 \%)$, eye secretions $(1,8 \%)$, and catheter tips $(0,9 \%)$. We identified seven species of Enterobacter including Enterobacter cloacae (54,1\%), Enterobacter sakazakii (22\%), Enterobacter aerogenes (15,6\%), Enterobacter agglomerans (2,8\%), Enterobacter cancerogenus (2,8\%), Enterobacter gergoviae $(1,8 \%)$ and Enterobacter amnigenus (0,9\%). 


\section{Open Access Journal of Microbiology \& Biotechnology}

Regarding the origin of bacteria, the intensive care unit was the most incriminated $(29,8 \%)$ followed by the pediatric unit ( $21,3 \%$ ), medicine unit ( $13,8 \%$ ), neonatalogy ( 12,8 $\%)$, emergency $(9,6 \%)$, surgery $(9,6 \%)$, gynecology ( $1,1 \%)$, ENT ( $1,1 \%)$ units and external (1,1\%).

\section{Antibiotic Susceptibility Profile}

The antibiotic susceptibility profile (Figure 1) revealed a high resistance to $\beta$-lactams with $100 \%$ imputed to amoxicillin and amoxicillin/clavulanic acid, $77 \%$ to cefuroxime, $67 \%$ toceftazidime, $49 \%$ to aztreonam and $46 \%$ toticarcillin. We also noted resistance to cotrimoxazole, gentamicin, nalidixic acid and colistin with respective frequencies of $58 \%, 45 \%, 42 \%$ and $30 \%$. In addition low resistance to carbapenems was observed with respective frequencies of $9 \%, 12 \%$ and $14 \%$ for imipenem, ertapenem and meropenem. Enterobacter species also showed a low resistance to amikacin with a frequencie of $13 \%$.

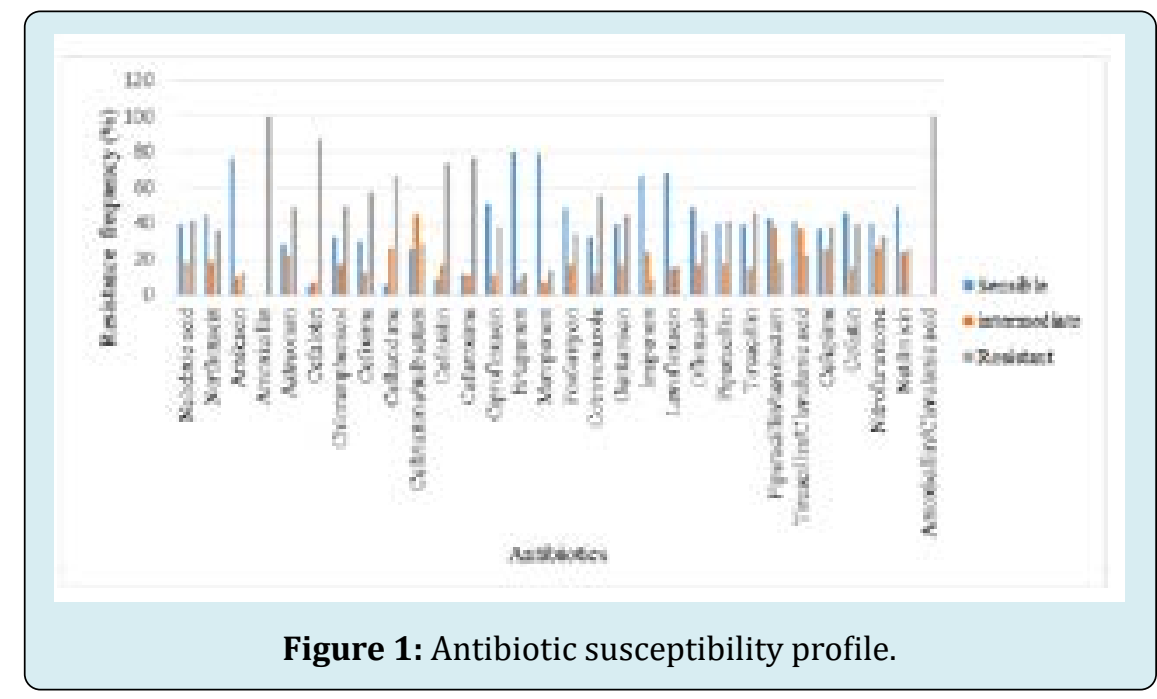

\section{Resistance Phenotypes}

Figure 2 shows the resistance strategy of the different families studied. The majority of the strains of Enterobacter spp were wild phenotypes in each family of antibiotics tested with values of $62.7 \%, 45.4 \%$ and $34.3 \%$ for quinolones, aminoglycosides and $\beta$-lactams respectively. Among the $\beta$ - lactam resistancephenotypes, extended spectrum $\beta$-lactamases, high-level cephalosporinases, high-level penicillinases and low-level penicillinases were observed with respective frequencies of $32.4 \%, 15,7 \%, 12 \%$ and
5.6\%. Regarding aminoglycosides, the phenotypes resistant gentamycin $(24.1 \%)$, resistant gentamycin-trobramycin $(6.5 \%)$, resistant gentamycin-trobramycin-netilmycin (13\%), resistant trobramycin-netilmicin-amikacin (4.6\%) and resistant gentamycin-netilmycin-amikacin $(6.5 \%)$ were observed. In addition, quinolone resistance phenotypes were observed with resistant nalixidic acid (17.3\%), resistant nalixidic acid-norfloxacin-pefloxacin (11.8\%) and nalixidic acid-norfloxacin-pefloxacin-ofloxacin-resistant phenotypes $(8,20 \%)$.

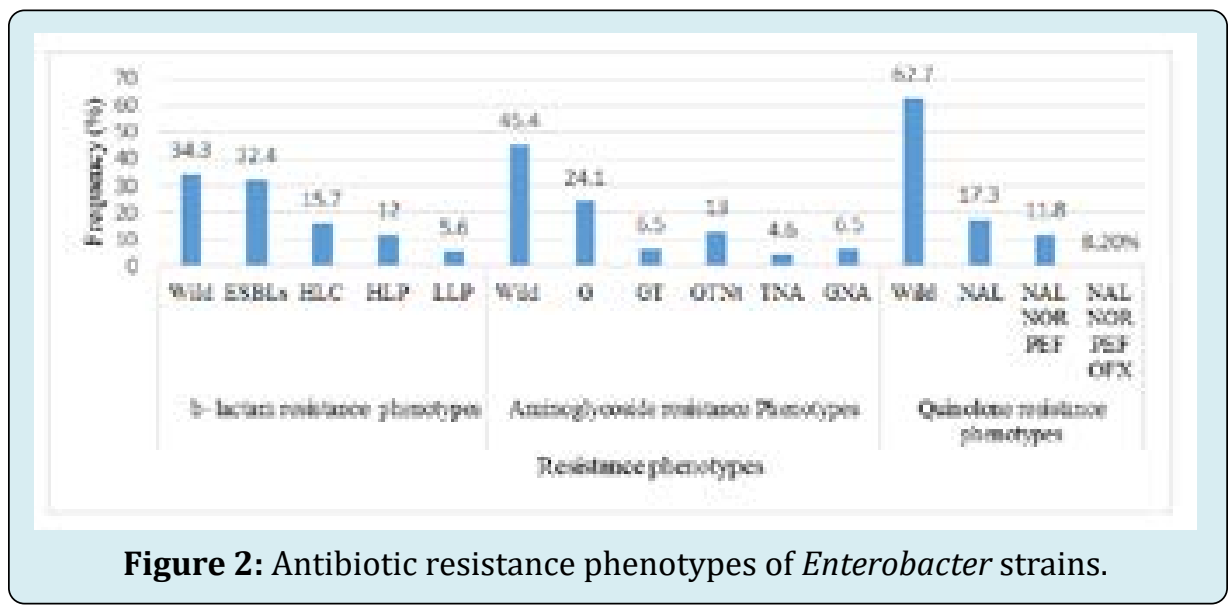

Kamga HG, et al. Evolution of Antibiotic Resistance in Enterobacter Spp Isolated at the Yaounde University Teaching Hospital from January 2008 to November 2019. J Microbiol Biotechnol 2021, 


\section{Open Access Journal of Microbiology \& Biotechnology}

ESBLs: extended spectrum $\beta$-lactamases; HLC: High-level cephalosporinases; HLP: High-level penicillinases; LLP: Low-level penicillinases; G: Gentamycin; T: Trobramycin; $\mathrm{N}$ : Netilmycin; A: amikacin; NAL: nalixidic acid; NOR: norfloxacin; PEF: pefloxacin; OFX: ofloxacin

\section{Evolution of Antibiotic Resistance}

Antibiotic resistance has varied from year to year between
2008 and 2019. Cephalosporins remain the most affected antibiotic with a resistance frequency varying from $40 \%$ to $80 \%$ with an optimum in 2012 (Figure 3). Enterobacter Spp showed a peak of resistance to the majority of antibiotic between 2011 and 2012 then between 2018 and 2019. Thus reflecting a re-emergence of resistance to these antibiotics. Penicillins, carbapenems and quinolones remain the most active antibiotics with the resistance frequencies below $37 \%$.

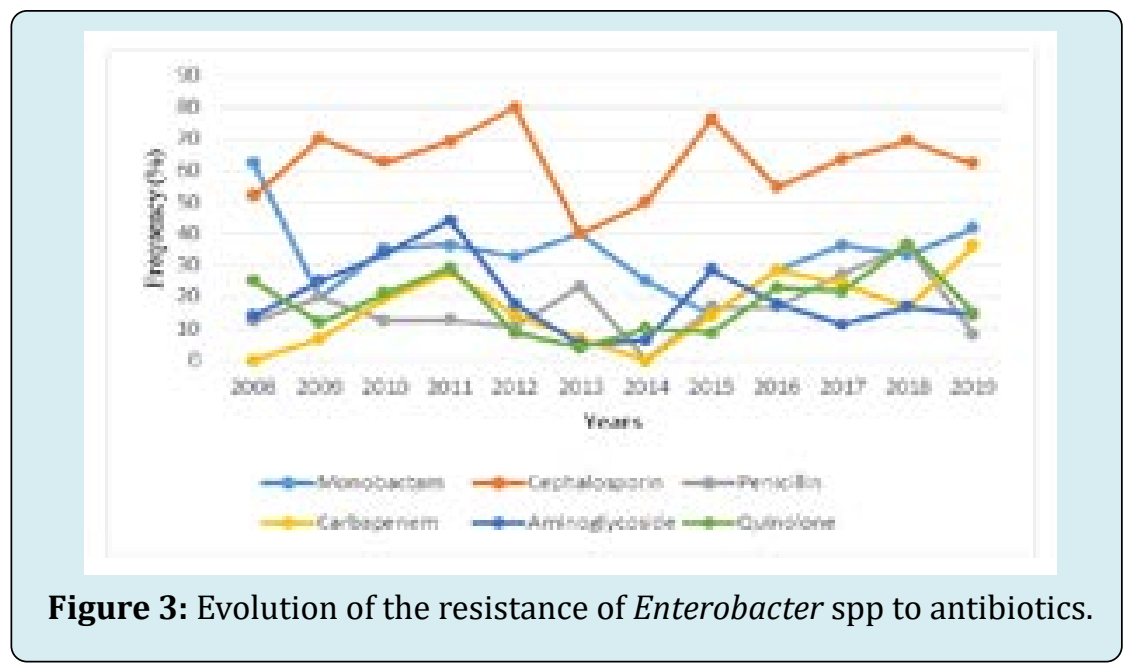

\section{Discussion}

Of the 109 strains isolated, the rates of Enterobacter infection for the female(50.5\%) and the male (49.5\%) were almost similar, indeed, sex is considered as a physiological criterion without influence on the infections linked to care [2]. Patients between 60 and 80 yearswere the most affected $(23.9 \%)$. This result is similar to those reported by previous studies conducted in Shanghai [12] and is due to the weakened immune system. For this study, blood was the most represented specimen (36.7\%). This result differs from that found by Gonsu et al. which presented urine as the most represented specimen [7]. This would be due to the fact that the species of the genus Enterobacter are ubiquitous. Enterobacter cloacae was most frequently isolated (54.1\%). This result is in agreement with those of several studies highlighting Enterobacter cloacae as the main species of this genus $[7,13]$. This study showed that intensive care unit presented the highest frequency of Enterobacter spp $(29,8 \%)$.Intensive care unit is known as a unit with several risk factors for infections. The study of Anthony, et al. [14] showed that many risks factors as surgery, urethral catherization, endotracheal intubation exposed patients to infections in this unit.

The study of the antibiotic resistance phenotypes indicated a total resistance to amoxicillin and amoxicillin/ clavulanic acid. Thusjustifying the wild phenotype. Resistance has been observed for cefuroxime (77\%), ceftazidine (67\%), cefixime (57\%) and aztreonam (49\%). The high resistance to first generation cephalosporins is due to a mutation in the AmpR repressor gene lead to high levels of AmpC production [15]. These results are slightly low compared to the resistance rate found in the study conducted in Algeria [2]. Low resistance was noted for meropenem (14\%), ertapenem (12\%) and imipenem (9\%). Previous studies presented a very high sensivity to carbapenems in Cameroon [7]. The resistance to carbapenem is due to ESBLs or porine alteration [16]. Resistance was noted to nalidixic acid (42\%), Ciprofloxacin (38\%) and norfloxacin (36\%). These percentages are higher than those found by Gonsu, et al. [7] in 2007, and are similar to those found by Khennouchi [2]. This shows an increase of resistance to quinolones recently. Resistance to quinolones is linked to the modification of the target enzyme or to the acquisitionof qnr-A and qnr-B genes [2,5]. Enterobacter strains showed low resistance to most aminoglycosides. Gentamicin showed an average resistance of $45 \%$. The resistance to gentamicin could be due to the mutation of genes in Enterobacter spp or to theproduction of an enzyme which inactivates aminoglycosides. Amikacin remains the most effective molecule with $87 \%$ of sensitive strains as reported by Boudjema [5]. However, we noted 30\% ofresistance to colistin which is a molecule that presented a high activity to Enterobacter. Resistance to this molecule is 


\section{Open Access Journal of Microbiology \& Biotechnology}

essentially linked to chromosomal mutations of the various genes leading to the modification of lipopolysaccharide charges which will prevent the attachment of colistin. Recently, colistin plasma genes, mcr- 1 and mcr- 2 have been described in several species [17]. The peak of resistance between 2018 and 2019 reflect an increase of resistance to the majority of antibiotics. Resistance to antibiotics is a growing fact and for which if nothing is done we will reach to a total resistance to all antibiotics for most bacteria.

\section{Conclusion}

This study presented various resistances of Enterobacter species to antibiotics during the last 12 years at the University Teaching Hospital of Yaounde. The cephalosporins were the most affected, but we also noted worrying resistance to many antibiotics. This study shows that many resistance phenotypes are present in Yaounde and antibiotic resistances have increased. These resistances require the establishment of a surveillance strategy in order to better detect and control the spread of multi-resistant strains and to adapt an adequate treatment regimen. It would therefore be important to establish a surveillance strategy in all the ten regions of Cameroon and in others countries to make an appropriate choice of first-line antibiotic therapy.

\section{References}

1. WHO, Organisation Mondiale de la Santé (2019) Résistance aux antibiotiques. World Health Organisation, Geneva, Switzerland.

2. Khennouchi NCEH (2016) Evaluation de l'antibioresistance du genre Enterobacter aux antibiotiques (Thèse de doctorat en médecine). Université Badji Mokhtar Annaba, Agérie, pp: 1-137.

3. Ebongue CO, Tsiazok MD, Mefo'o JPN, Ngaba GP, Beyiha G, et al. (2015) Evolution de la résistance aux antibiotiques des entérobactéries isolées à l'Hôpital Général de Douala de 2005 à 2012. The Pan African Medical Journal 20(1): 1-11.

4. Samuel NA, Marie COA (2013) Antibiotiques et résistance bactérienne : un sujet de préoccupation. Health Sci Dis 14(4): 1-2.

5. Boudjema NSD (2015) Etude multicentrique de la résistance aux antibiotiques chez Enterobacter cloacae. Thèse de doctorat en biologie, Université Abou Bekr Belkaid-Tlemcen, Algérie, pp: 1-143.

6. Pontiès $\mathrm{V}$, Colomb Cotinat $\mathrm{M}$, Soing Altach $\mathrm{S}$, Assouvie L, Berger Carbonne A (2018) Bilan national des signalements d'infection / colonisation à Enterobacter cloacae chez les nouveau-nés hospitalisés en France, 2012-2017. Bulletin national CPias pp: 10: 1-5.

7. Gonsu HK, Kamgue S, Toukam M, Lyonga E (2009) Diversity and distribution of biotypes and antibiotic resistance phenotypes of Enterobacter ssp. Isolated from patients in Yaoundé, Cameroon. Health Sci Dis 10(2): 1-7.

8. Nouetchognou JS, Ateudjieu J, Jemea B, Mesumbe EN, Mbanya DNS (2016) Surveillance of nosocomial infections in the Yaounde University Teaching Hospital, Cameroon. BMC Res Notes 9(1): 505.

9. EUCAST (2019) Comité de l'antibiogramme de la Société Française de Microbiologie. European society of Clinical Microbiology and Infectious Diseases pp: 1-144.

10. CLSI (2011) Performance Standards for Antimicrobial Susceptibility Testing, European Commitee on Antimicrobial Susceptibility Testing Breakpoint tables for interpretation of MICs and zone diameters. M100-S21, pp: 1-172.

11. Boutal H (2017) Développement et validation de test de détection rapide de la résistance aux antibiotiques (Thèse de doctoraten biochimie et biologie structurale). Université Paris-Saclay, France, pp: 1-66.

12. Wang S, Xiao SZ, Gu FF, Tang J, Guo XK, et al. (2017) Antimicrobial susceptibility and molecular epidemiology of clinical Enterobacter cloacae bloodstream isolates in Shanghai, China. PloS One 12(12): e0189713.

13. Boukerouaz A, Benmehidi R (2017) Profil bactériologique des bactériémies à bacilles Gram négatif. (Mémoire de Master Microbiologie générale et Biologie Moleculaire des Microorganisme, Université des Frères Mentouri Constantine Algérie), pp: 1-59.

14. Wuafor AA, Ogunsola FT, Oladele RO, Oduyebo OO, DesaluI, et al. (2016) Incidence, Clinical Outcome and Risk Factors of Intensive Care Unit Infections in the Lagos University Teaching Hospital (LUTH), Lagos, Nigeria. PLoS One 11(10): e0165242.

15. Guérin F (2015) Infections à Enterobacter cloacae complex: Résistance aux antibiotiques et traitement. Journal des Anti-infectieux 17(3): 79-89.

16. Grall N, Andremont A, Armand Lefèvre L (2011) Résistance aux carbapénèmes: Vers une nouvelle impasse ? Journal des Anti-infectieux 13(2): 87-102. 


\section{Open Access Journal of Microbiology \& Biotechnology}

17. Dortet L, Bonnin R, Jousset A, Gauthier L, Naas T (2016) Émergence de la résistance à la colistine chez les entérobactéries: Une brèche dans le dernier rempart contre la pan-résistance! Journal des Anti-infectieux 18(4): 139-159. 